Research Article

\title{
Tuning Electronic Properties of GaSe/Silicane Van der Waals Heterostructure by External Electric Field and Strain: A First-Principle Study
}

\author{
Gang Xu $(\mathbb{D})$ and Hao Lei \\ Department of Physics, Chongqing Three Gorges University, Wanzhou 404100, China \\ Correspondence should be addressed to Gang Xu; 20040026@sanxiau.edu.cn
}

Received 20 January 2021; Revised 10 February 2021; Accepted 25 February 2021; Published 11 March 2021

Academic Editor: Gary Wysin

Copyright (c) 2021 Gang $\mathrm{Xu}$ and Hao Lei. This is an open access article distributed under the Creative Commons Attribution License, which permits unrestricted use, distribution, and reproduction in any medium, provided the original work is properly cited.

\begin{abstract}
The electronic structure of GaSe/silicane $(\mathrm{GaSe} / \mathrm{SiH})$ van der Waals (vdW) heterostructure in response to a vertical electric field and strain was studied via first-principle calculations. The heterostructure had indirect band gap characteristics in the range $[-1.0$, $-0.4] \mathrm{V} / \AA$ and direct band gap features in the range $[-0.3,0.2] \mathrm{V} / \AA$. Furthermore, a type-II to type-I band alignment transition appeared at -0.7 and $-0.3 \mathrm{~V} / \AA$. Additionally, the $\mathrm{GaSe} / \mathrm{SiH}$ vdW heterostructure had a type-II band alignment under strain, but an indirect to direct band gap semiconductor transition occurred at $-3 \%$. These results indicated that the $\mathrm{GaSe} / \mathrm{SiH}$ vdW heterostructure may have applications in novel nanoelectronic and optoelectronic devices.
\end{abstract}

\section{Introduction}

Since the successful exfoliation of graphene by Novoselov et al. in 2004, there has been intense research interest in twodimensional (2D) materials in the field of condensed matter physics [1]. A large number of 2D materials such as silicene [2-5], germanane [6], transition metal halides [7], transition metal chalcogenides [8-10], transition metal dichalcogenides (TMDs) [11-15], and the lesser known family of semiconducting monolayer metal monochalcogenides (MX, $\mathrm{M}=\mathrm{Ga}, \mathrm{X}=\mathrm{S}$, Se) [11-19] have been reported. However, each $2 \mathrm{D}$ material has its own advantages and disadvantages; hence, one material cannot be broadly applied to all fields. For example, the zero-band gap characteristics of graphene limit its applications in switching devices; in contrast, $\mathrm{BN}$ is a typical insulator with a very large band gap [20-22]. To overcome this issue, recent studies have reported that two different 2D materials can be stacked vertically to construct a van der Waals (vdW) heterostructure that not only retains the excellent characteristics of the original 2D materials but may also present novel physical properties because of the
vdW forces between the two layers [23-26]. This approach has opened up new ways to study nanoelectronics and optoelectronic devices; hence, it has become a field of intense research interest.

Monolayer GaSe is a member of the 2D MX family that has successfully been fabricated via chemical vapor deposition [27], pulsed laser deposition [28], and micromechanical cleavage techniques [29], and it has subsequently been widely studied [30-33]. Reports have theoretically demonstrated that the electronic structures, transport, and optical properties of GaSe monolayer are sensitive to applied electric fields and strains [30-32]. Furthermore, a number of nanoelectronic and optoelectronic devices based on monolayer GaSe with high photoresponse and on/off ratios have been successfully fabricated [31, 32]. These theoretical and experimental studies predicted that monolayer GaSe is a promising candidate for nanoelectronic and optoelectronic applications. Silicane ( $\mathrm{SiH})$, a fully hydrogenated silicene, has the same structure as silicene, but has a large band gap of $\sim 2.93 \mathrm{eV}$ [34-36]. Numerous studies have demonstrated that the electronic band structure of $\mathrm{SiH}$ can be tuned via a 
uniform tensile strain and applied electric field [36-38]. Moreover, Low et al. [39] found that a SiH n-MOSFET offers a relatively better $\mathrm{ON}$-current performance than transistors made of germanane and 2D TMD materials. These characteristics make $\mathrm{SiH}$ a promising candidate for electronic and optoelectronic devices.

In addition, a large number of $\mathrm{vdW}$ heterostructures have been experimentally synthesized and theoretically proposed in recent years, including $\mathrm{BN} /$ graphene [23], $\mathrm{WS}_{2}$ / $\mathrm{MoS}_{2}$ [40], and $\mathrm{MoS}_{2} / \mathrm{GaN}$ [41]. In particular, the combinations between $\mathrm{SiH}$ and $\mathrm{GaSe}$ with other 2D materials, including GaSe/MoS 2 [17], GaSe/graphene, SiH/graphane, and $\mathrm{SiH} /$ hydrogenated hexagonal boron nitride have also received significant interest because of the preservation of their intriguing electronic properties and enhancement of their optical properties [42, 43]. For example, Jiang et al. [42] theoretically found that the band gaps of the $\mathrm{SiH} /$ graphane and $\mathrm{SiH} /$ hydrogenated hexagonal boron nitride could be tuned from a semiconductor to a metal under an applied electric field. Meanwhile, Pham et al. [44] demonstrated that the GaSe/ $\mathrm{MoS}_{2}$ vdW heterostructure is an indirect band gap semiconductor with a band gap of $1.91 \mathrm{eV}$ and a type-II band alignment. Furthermore, many research groups have successfully synthesized atomic layer $\mathrm{GaSe} / \mathrm{MoS}_{2}$ van der Waals heterostructures and applied them for photoresponse modulation and as photodiodes $[45,46]$. These reports indicate that $\mathrm{GaSe}$ - and $\mathrm{SiH}$-based vdW heterostructures are promising $2 \mathrm{D}$ semiconductors with a high potential for novel applications in electronic and optoelectronic nanodevices. However, to date, there have been no reports focusing on the combination of $\mathrm{GaSe}$ with $\mathrm{SiH}$ monolayers and its electronic structure, as well as the electric field and strain effects. Therefore, this work investigated the design of GaSe/ $\mathrm{SiH}$ vdW heterostructures and studied their electronic structures via first-principle calculations. The electric field and strain effects on the electronic structures of $\mathrm{GaSe} / \mathrm{SiH}$ vdW heterostructures were also considered.

\section{Computational Methods}

The optimized structures and electronic structures of GaSe/ $\mathrm{SiH}$ vdW heterostructures were obtained via first-principles calculations based on the density functional theory. The generalized gradient approximation of the Perdew-Burke-Ernzerhof functional $[47,48]$ was used for crystal structure relaxation, which was a part of the SIESTA code $[49,50]$. The norm-conserving pseudopotentials were used to solve the Kohn-Sham equations. The cutoff energy was set at 100 Hatree, and a Brillouin zone sampling $K$-mesh of $9 \times 9 \times 1$ was chosen. To obtain more accurate results, a $K$ mesh of $11 \times 11 \times 1$ was adopted for the energy band structure calculations. For relaxation, the energy criterion was set as $10^{-4} \mathrm{eV}$, and the residual force was smaller than $-0.05 \mathrm{eV} / \AA$. The vacuum layer thickness was set to $20 \AA$ to avoid other interactions with neighboring layers. Importantly, the long-ranged vdW interaction was required to hold the vdW heterostructure together. Here, the vdW-DF3 functional was chosen to describe long-range electron correlation effects [51].

\section{Results and Discussion}

3.1. Optimized Structures and Formation Energy. First, we optimized the original monolayer $\mathrm{SiH}$ and GaSe. The optimized lattice constants of $\mathrm{SiH}$ and GaSe were 3.889 and $3.847 \AA$, which are consistent with pre-existing theoretical and experimental values [35, 37, 38, 52-54]. Therefore, we built the $\mathrm{GaSe} / \mathrm{SiH}$ vdW heterostructure using one unit cell of $\mathrm{SiH}$ and $\mathrm{GaSe}$. The lattice mismatch was defined as

$$
2 \times \frac{\left(a_{1}-a_{2}\right)}{\left(a_{1}+a_{2}\right)} \times 100 \%
$$

where $a_{1}$ and $a_{2}$ represent the lattice constants of $\mathrm{SiH}$ and $\mathrm{GaSe}$, respectively. The calculated result was only $\sim 1.1 \%$, indicating that the $\mathrm{GaSe} / \mathrm{SiH}$ vdW heterostructure could be easily fabricated with very little interfacial stress. Hence, we built six possible heterostructure stacking configurations to find the ground structure, as shown in Figures 1(a)-1(f)) and labeled as model I to model VI.

To analyze the vdW interactions between $\mathrm{SiH}$ and the GaSe layers, the formation energy $\left(E_{f}\right)$ was defined as

$$
E_{f}=E_{\mathrm{SiH} / \mathrm{GaSe}}-E_{\mathrm{SiH}}-E_{\mathrm{GaSe}},
$$

where $E_{f}$ represents the formation energy of the $\mathrm{GaSe} / \mathrm{SiH}$ vdW heterostructures and $\mathrm{E}_{\mathrm{SiH} / \mathrm{GaSe}}, \mathrm{E}_{\mathrm{SiH}}$, and $\mathrm{E}_{\mathrm{GaSe}}$ represent the total energy of the $\mathrm{GaSe} / \mathrm{SiH}$ vdW heterostructures, $\mathrm{SiH}$, and $\mathrm{GaSe}$ monolayer, respectively. The calculated values of $E_{f}$ are listed in Table 1. All the calculated $E_{f}$ values were negative, indicating that all the six stacking configurations were stable from an energy perspective. Among them, the conformation model $\mathrm{V}$ was the ground structure because it had the lowest formation energy. In addition, we listed the interface distance $(\Delta$, as shown in Figure 1(f)) in Table 1. The conformation model $V$ has the smallest distance, indicating that the conformation model $\mathrm{V}$ was the ground structure. Therefore, in the following section, we only focused on conformation model $\mathrm{V}$.

Next, we calculated the band structures of $\mathrm{SiH}, \mathrm{GaSe}$, and the $\mathrm{GaSe} / \mathrm{SiH}$ vdW heterostructures, as shown in Figure 2. The conduction band minimum (CBM) of $\mathrm{SiH}$ was located at the $M$ point of the two-dimensional hexagonal Brillouin zone, while the valence band maximum (VBM) was located at the $\Gamma$ point; this indicated that the pristine $\mathrm{SiH}$ is an indirect band gap semiconductor, with a corresponding band gap of $2.184 \mathrm{eV}$, which is in line with the results of the papers by Wang et al. and Guzmán-Verri et al. $[38,55,56]$. The CBM of GaSe was located at the $\Gamma$ points, while the VBM was on the line of $\Gamma-\mathrm{M}$ and was close to the $\Gamma$ points; this indicated the formation of an indirect semiconductor with a band gap of $1.871 \mathrm{eV}$. The calculated band gap value was similar to the theoretical values of 1.60 and $1.77 \mathrm{eV}$ obtained from the PBE method [52-54]. The band structures for the GaSe/SiH vdW heterostructure are shown in Figure 2(c). Both the VBM and CBM of the GaSe/ $\mathrm{SiH}$ vdW heterostructure are situated at the $\Gamma$ points, indicating a direct semiconductor with a band gap of $0.558 \mathrm{eV}$. Furthermore, the VBM was predominantly derived from the $\mathrm{SiH}$ layer, and the $\mathrm{CBM}$ had a contribution 

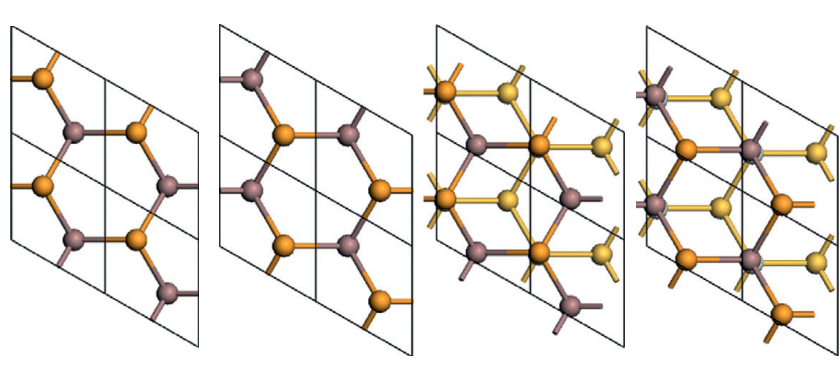

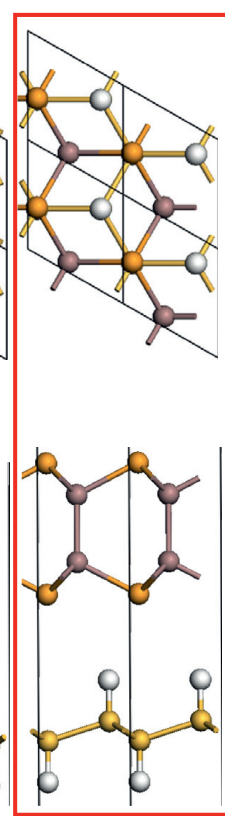

(e)

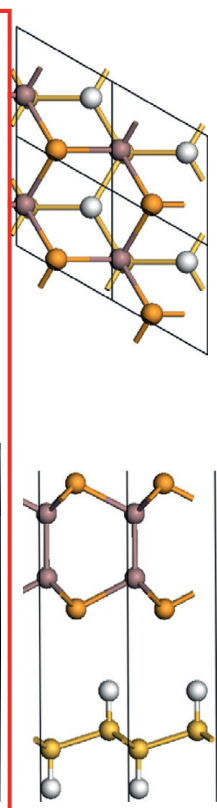

(f)

Figure 1: The lattice structure of GaSe/SiH vdW heterostructure: (a) model I, (b) model II, (c) model III, (d) model IV, (e) model V, and (f) model VI. The upper part is for top view, and the lower part is for side view. The $\mathrm{Ga}, \mathrm{Se}, \mathrm{Si}$, and $\mathrm{H}$ atoms are represented by brown, yellow, golden yellow, and white balls, respectively.

TABLE 1: The formation energy $\left(E_{f}\right)$ and interface distance $(\Delta)$ for the six stacking conformations.

\begin{tabular}{lcccccc}
\hline Model & I & II & III & IV & V & VI \\
\hline$E_{f} / \mathrm{eV}$ & -0.855 & -0.838 & -0.838 & -0.856 & -0.857 & -0.856 \\
$\Delta / \AA$ & 3.217 & 3.233 & 3.218 & 3.233 & 3.164 & 3.189 \\
\hline
\end{tabular}

from the GaSe layer, i.e., a representative type-II band alignment [57-60]. To understand the electronic structures of the $\mathrm{GaSe} / \mathrm{SiH}$ vdW heterostructures in detail, we plotted the energy band alignment of the GaSe/SiH vdW heterostructure, as shown in Figure 2(d). The band edges of $\mathrm{SiH}$ and GaSe were $(1.875,-0.313) \mathrm{eV}$ and $(0.245,-1.319) \mathrm{eV}$, respectively. The CBM and VBM of the GaSe were lower than those of the $\mathrm{SiH}$, which further verified the type-II band alignment of the $\mathrm{GaSe} / \mathrm{SiH}$ vdW heterostructure. In addition, we found that there was a small discrepancy between our study and previous studies because we used a different software package. Furthermore, we also found that these band gaps were smaller than the values obtained via the HSE method because the PBE method underestimated the band gap. The standard PBE functional enabled the prediction of the correct trend for the band structures, properly demonstrating their physical mechanisms. Hence, to save on computational load, we used the PBE method to perform electronic structure calculations for the $\mathrm{GaSe} / \mathrm{SiH}$ vdW heterostructures under an applied electric field and strain.

3.2. External Electric Field Effect. Previously reported theoretical and experimental results showed that an applied electric field can significantly affect the band structures of the vdW heterostructure, so we studied the electronic structures of the $\mathrm{GaSe} / \mathrm{SiH}$ vdW heterostructure under a vertical electric field $[38,61-66]$. The electric field was perpendicular to the $\mathrm{GaSe} / \mathrm{SiH}$ vdW heterostructure, and the positive direction of the electric field was from $\mathrm{SiH}$ towards GaSe. The range for the external electric field was $[-1.0,1.0] \mathrm{V} / \AA$, and the step length was $0.1 \mathrm{~V} / \AA$. The influence of the electric field on the band edges, band gap, and band structures of the $\mathrm{GaSe} / \mathrm{SiH}$ vdW heterostructure is shown in Figure 3 and Figure S1. The VBM derived from $\mathrm{GaSe}$ and the CBM derived from $\mathrm{SiH}$ were in the range of $[-1.0,-0.7] \mathrm{V} / \AA$, both the $\mathrm{VBM}$ and $\mathrm{CBM}$ derived from GaSe were in the range of $[-0.6,-0.4] \mathrm{V} / \AA$, and the VBM derived from $\mathrm{SiH}$ and the $\mathrm{CBM}$ derived from GaSe were in the range of $[-0.3,0.2] \mathrm{V} / \AA$. These results indicated that the $\mathrm{GaSe} / \mathrm{SiH}$ vdW heterostructure had a type-II band alignment in the range of $[-1.0,-0.7]$ and $[-0.3,0.2] \mathrm{V} / \AA$, while it had a type-I band alignment in the range of $[-0.6,-0.4]$ $\mathrm{V} / \mathrm{A}$. Moreover, the VBM lay on the $\Gamma-\mathrm{M}$ line and was close to the $\Gamma$ point in the range of $[-1.0,-0.4] \mathrm{V} / \AA$ and was located at the $\Gamma$ points in the range of $[-0.3,0.2] \mathrm{V} / \AA$. The CBM was located at the $M$ points in the range of $[-1.0$, $-0.6] \mathrm{V} / \AA$ and was located at the $\Gamma$ points in the range of $[-0.5,0.2] \mathrm{V} / \AA$. These numerical results demonstrated that the $\mathrm{GaSe} / \mathrm{SiH}$ vdW heterostructure was an indirect band gap semiconductor in the range of $[-1.0,-0.4] \mathrm{V} / \AA$, while it was a direct band gap semiconductor in the range of $[-0.3$, 0.2] V/Å. Furthermore, a metal-semiconductor phase transition occurred at $0.3 \mathrm{~V} / \AA$. In summary, the $\mathrm{GaSe} / \mathrm{SiH}$ $\mathrm{vdW}$ heterostructure was an indirect band gap semiconductor with a type-II band alignment in the range of $[-1.0$, -0.7] V/A, an indirect band gap semiclonductor with a type-I band alignment in the range of $[-0.6,-0.4] \mathrm{V} / \AA$, a 


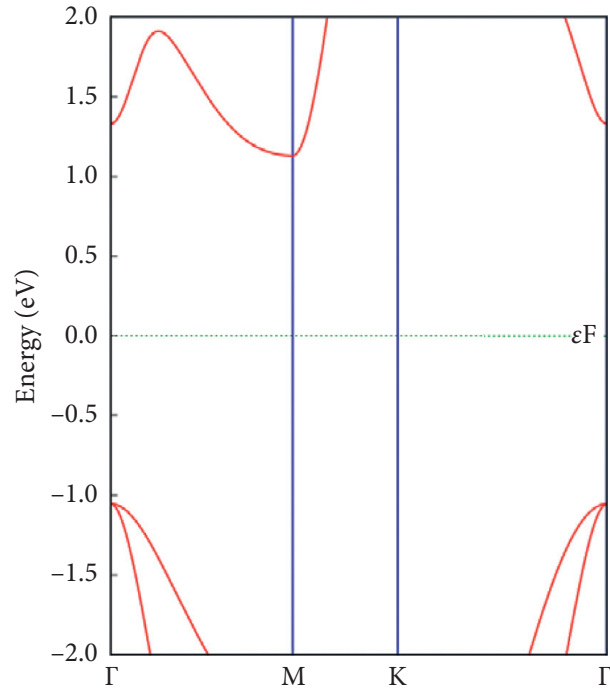

(a)

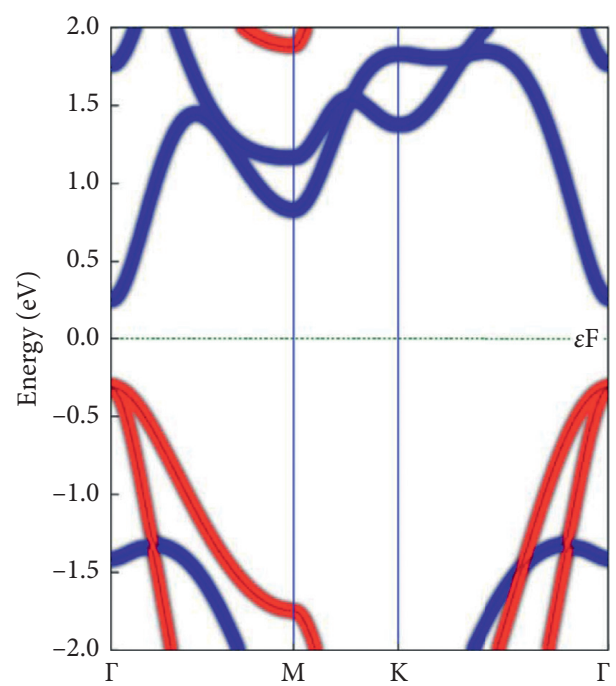

(c)

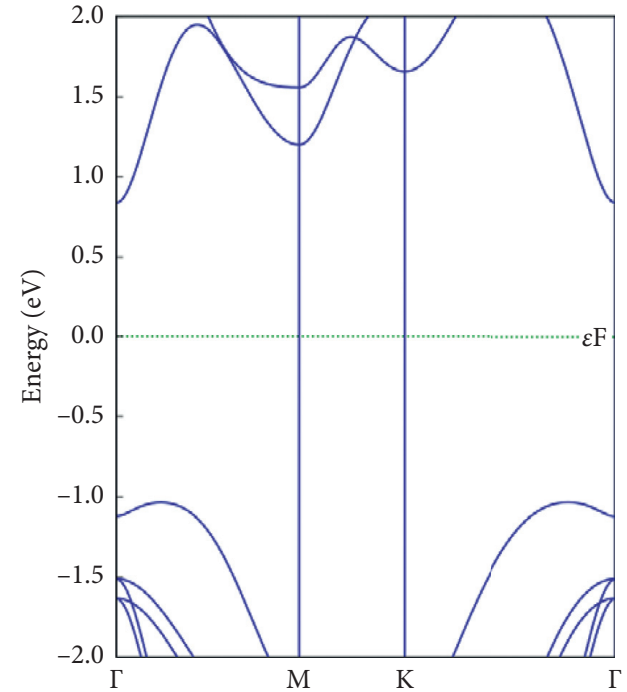

(b)

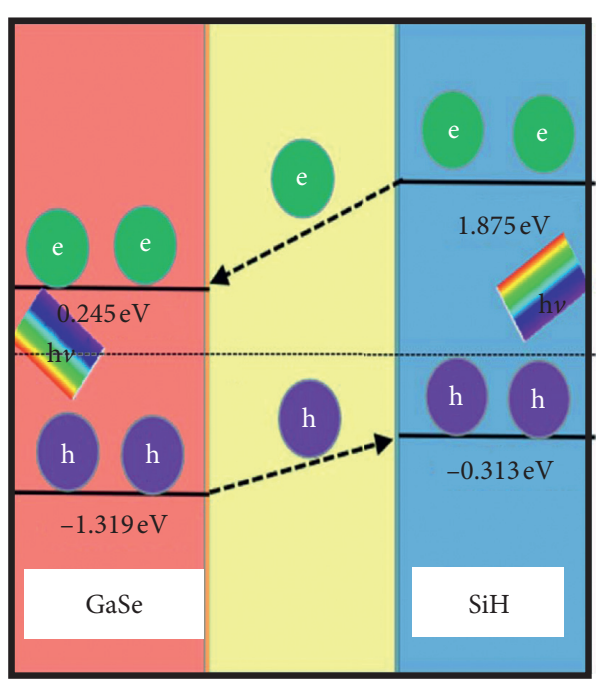

(d)

Figure 2: The band structures of (a) $\mathrm{SiH}$, (b) GaSe, and (c) GaSe/SiH vdW heterostructure. The blue lines represent the GaSe, while the red lines represent the $\mathrm{SiH}$. (d) The band offset of the GaSe/SiH vdW heterostructure.

direct band gap semiconductor with a type-II band alignment in the range of $[-0.3,0.2] \mathrm{V} / \AA$, and it then changed to the metallic state. These results indicated that the $\mathrm{GaSe} / \mathrm{SiH}$ vdW heterostructure had a variable band structure under an applied electric field.

3.3. Strain Effect. Strain is known to also affect the electronic structures of the vdW heterostructure [38, 63-68]. Here, we studied the effect of inplane biaxial strain on the electronic structures of the $\mathrm{GaSe} / \mathrm{SiH}$ vdW heterostructure. The inplane biaxial strain on the $\mathrm{GaSe} / \mathrm{SiH}$ vdW heterostructure was emulated by changing the crystal lattice parameters and then calculating in accordance with the following formula:

$$
\Theta=\left[\left(a-a_{0}\right) / a_{0}\right] \times 100 \%,
$$

where $a_{0}$ and $a$ are the crystal lattice parameters under the unstrained and strained conditions, respectively. The external strain was applied in the range of $-5 \%$ to $5 \%$ in steps of $1 \%$. The strain effects on the band edges, band gap, and electronic structures of the $\mathrm{GaSe} / \mathrm{SiH}$ vdW heterostructure are shown in Figure 4 and Figure S2. The band gap increased with an increasing strain in the range of $[-5 \%,-4 \%]$ and then decreased with increasing strain in the range of $[-3 \%$, $5 \%]$. The VBM was located at the $\Gamma$ points over the whole range, while the CBM was located at the $M$ points in the range of $[-5 \%,-4 \%]$ and at the $\Gamma$ points in the range of $[-3 \%$, 5\%]. Furthermore, the VBM was derived from $\mathrm{SiH}$, and the CBM was derived from GaSe. Therefore, the $\mathrm{GaSe} / \mathrm{SiH}$ vdW heterostructure had a type-II band alignment under strain. The above results demonstrated that the $\mathrm{GaSe} / \mathrm{SiH}$ vdW heterostructure is an indirect band gap semiconductor with 


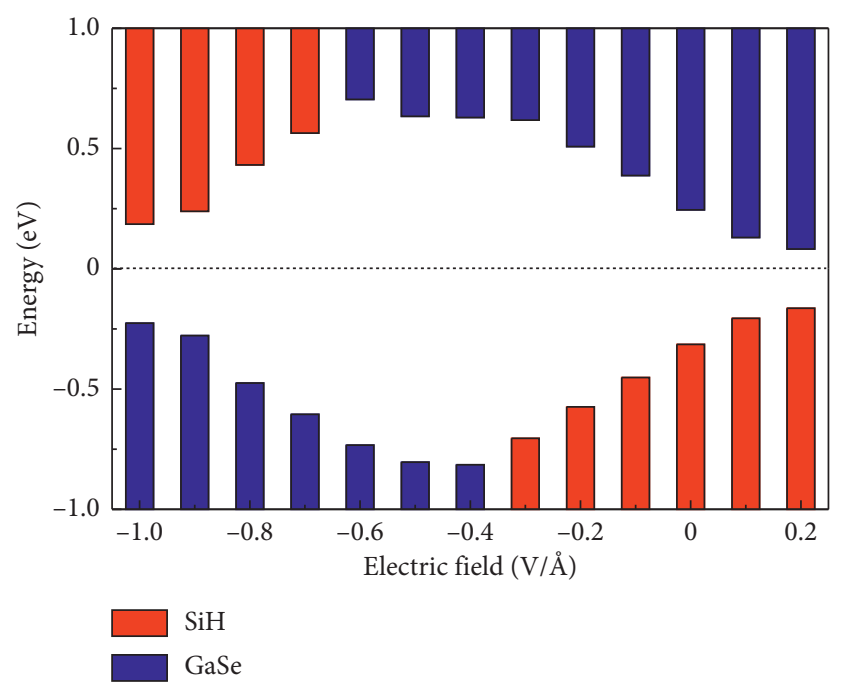

(a)

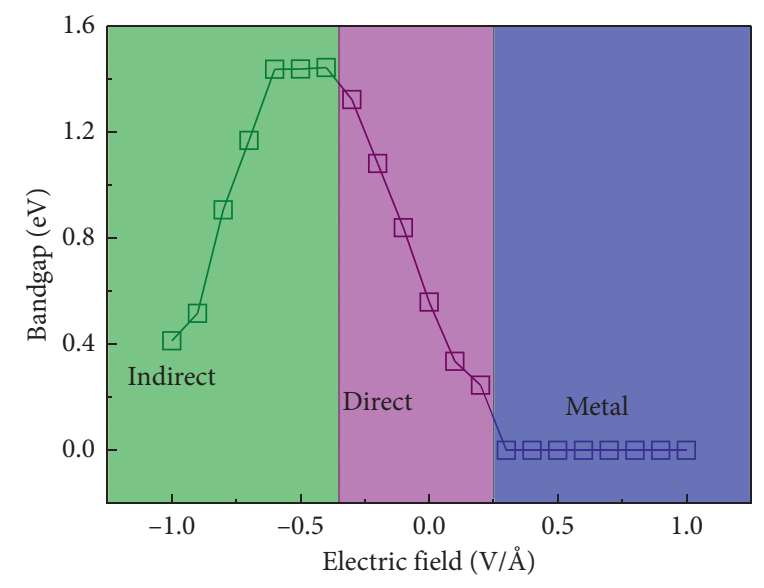

(b)

Figure 3: The (a) band edge and (b) band gap as a function of the external external field strength in the GaSe/SiH vdW heterostructure.

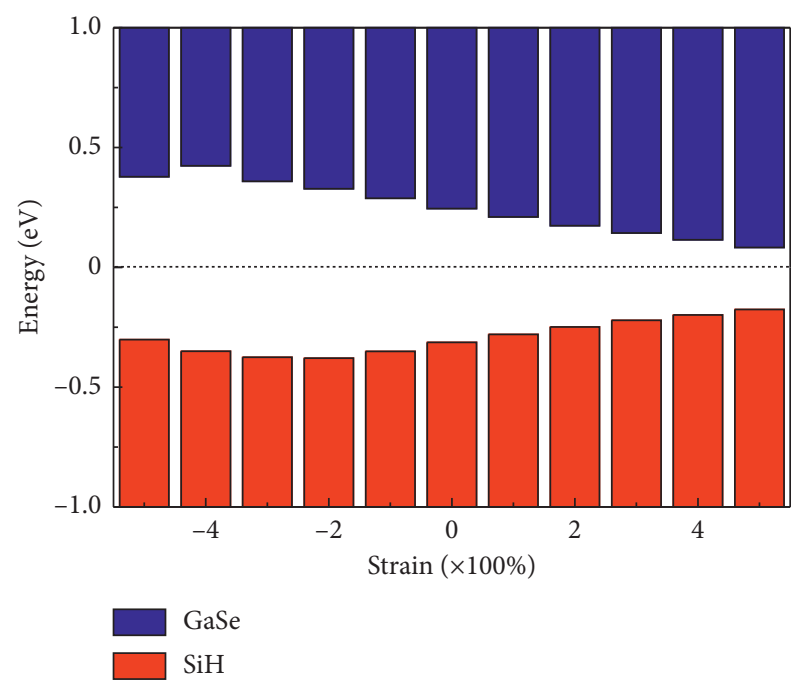

(a)

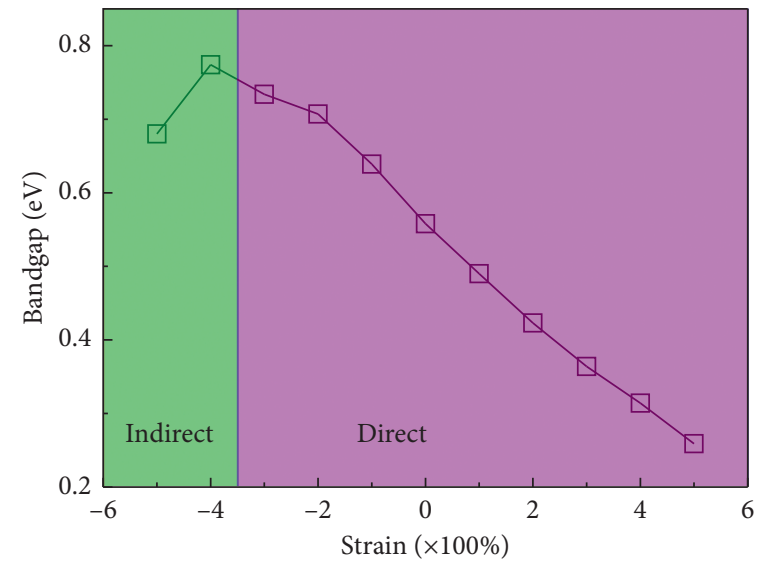

(b)

Figure 4: The (a) band edge and (b) band gap as a function of the strain strength in the GaSe/SiH vdW heterostructure.

a type-II band alignment in the range of [ $-5 \%,-4 \%]$, while it is a direct band gap semiconductor with a type-II band alignment in the range of $[-3 \%, 5 \%]$. The results of this work indicate that the electronic structures of the $\mathrm{GaSe} / \mathrm{SiH}$ vdW heterostructure can be effectively regulated by strain.

\section{Conclusion}

In summary, the electronic structures of the GaSe/SiH vdW heterostructure under an applied electric field and strain were determined via first-principles calculations. We observed that the $\mathrm{GaSe} / \mathrm{SiH}$ vdW heterostructure was an indirect band gap semiconductor with a type-II band alignment in the range of $[-1.0,-0.7] \mathrm{V} / \AA$, an indirect band gap semiconductor with a type-I band alignment in the range of $[-0.6,-0.4] \mathrm{V} / \AA$, a direct band gap semiconductor with a type-II band alignment in the range of $[-0.3,0.2] \mathrm{V} / \AA$, and it then transitioned to the metallic state. Furthermore, the $\mathrm{GaSe} / \mathrm{SiH}$ vdW heterostructure was an indirect band gap semiconductor with a type-II band alignment in the range of $[-5 \%,-4 \%]$, while it was a direct band gap semiconductor with a type-II band alignment in the range of $[-3 \%, 5 \%]$. The results indicated that the tunable band gap of the $\mathrm{GaSe} / \mathrm{SiH}$ $\mathrm{vdW}$ heterostructures provides a promising avenue for the fabrication of novel nanoelectronic and optoelectronic devices.

\section{Data Availability}

No data were used to support this study. 


\section{Conflicts of Interest}

The authors declare no conflicts of interest.

\section{Acknowledgments}

The authors thank Arun Paraecattil, Ph.D., from Liwen Bianji, Edanz Editing, China (http://www.liwenbianji.cn/ac), for editing the English text of a draft of this manuscript.

\section{Supplementary Materials}

Figure 1S: band structures of the $\mathrm{GaSe} / \mathrm{SiH}$ vdW heterostructure under applied electric fields with steps of $0.1 \mathrm{~V} / \AA$. The upper levels from left to right are -1 to $-0.1 \mathrm{~V} / \AA$, while the lower levels from left to right are 0.1 to $1 \mathrm{~V} / \AA$. The blue lines represent the GaSe, while the red lines represent the $\mathrm{SiH}$. Figure 2S: band structures of the $\mathrm{GaSe} / \mathrm{SiH}$ vdW heterostructure under inplane biaxial strains with steps of $1 \%$; from left to right, the strains are $-5 \%$ to $5 \%$. The blue lines represent the GaSe, while the red lines represent the $\mathrm{SiH}$. (Supplementary Materials)

\section{References}

[1] K. S. Novoselov, A. K. Geim, S. V. Morozov et al., "Electric field effect in atomically thin carbon films," Science, vol. 306, no. 5696, pp. 666-669, 2004.

[2] P. Vogt, P. De Padova, C. Quaresima et al., "Silicene: compelling experimental evidence for graphenelike two-dimensional silicon," Physical Review Letters, vol. 108, Article ID 155501, 2012.

[3] L. Meng, Y. Wang, L. Zhang et al., "Buckled silicene formation on $\operatorname{Ir}(111)$," Nano Letters, vol. 13, no. 2, pp. 685-690, 2013.

[4] C.-L. Lin, R. Arafune, K. Kawahara et al., "Structure of silicene grown on Ag (111)," Applied Physics Express, vol. 5, Article ID 045802, 2012.

[5] S. Fathipour, M. Remskar, A. Varlec et al., "Synthesized multiwall MoS2 nanotube and nanoribbon field-effect transistors," Applied Physics Express, vol. 106, Article ID 022114, 2015.

[6] R. K. Ghosh, M. Brahma, and S. Mahapatra, "Germanane: a low effective mass and high bandgap 2-D channel material for future FETs," IEEE Transactions on Electron Devices, vol. 61, pp. 2309-2315, 2014.

[7] C. Huang, J. Zhou, H. Wu, K. Deng, P. Jena, and E. Kan, "Quantum anomalous Hall effect in ferromagnetic transition metal halides," Physical Review B, vol. 95, Article ID 045113, 2017.

[8] T. Heine, "Transition metal chalcogenides: ultrathin inorganic materials with tunable electronic properties," Accounts of Chemical Research, vol. 48, no. 1, pp. 65-72, 2015.

[9] Y. Jung, Y. Zhou, and J. J. Cha, "Intercalation in two-dimensional transition metal chalcogenides," Inorganic Chemistry Frontiers, vol. 3, no. 4, pp. 452-463, 2016.

[10] D. Yoo, M. Kim, S. Jeong, J. Han, and J. Cheon, "Chemical synthetic strategy for single-layer transition-metal chalcogenides," Journal of the American Chemical Society, vol. 136, no. 42, pp. 14670-14673, 2014.

[11] D. Jariwala, V. K. Sangwan, L. J. Lauhon, T. J. Marks, and M. C. Hersam, "Emerging device applications for semiconducting two-dimensional transition metal dichalcogenides," ACS Nano, vol. 8, no. 2, pp. 1102-1120, 2014.
[12] K. F. Mak and J. Shan, "Photonics and optoelectronics of 2D semiconductor transition metal dichalcogenides," Nature Photonics, vol. 10, no. 4, p. 216, 2016.

[13] S. Manzeli, D. Ovchinnikov, D. Pasquier, O. V. Yazyev, and A. Kis, "2D transition metal dichalcogenides," Nature Reviews Materials, vol. 2, Article ID 17033, 2017.

[14] Q. H. Wang, K. Kalantar-Zadeh, A. Kis, J. N. Coleman, and M. S. Strano, "Electronics and optoelectronics of two-dimensional transition metal dichalcogenides," Nature Nanotechnology, vol. 7, no. 11, p. 699, 2012.

[15] X. Xu, W. Yao, D. Xiao, and T. F. Heinz, "Spin and pseudospins in layered transition metal dichalcogenides," Nature Physics, vol. 10, no. 5, pp. 343-350, 2014.

[16] T. Cao, Z. Li, and S. G. Louie, "Tunable magnetism and halfmetallicity in hole-doped monolayer GaSe," Physical Review Letters, vol. 114, Article ID 236602, 2015.

[17] X. Li, M.-W. Lin, J. Lin et al., "Two-dimensional GaSe/MoSe ${ }_{2}$ misfit bilayer heterojunctions by van der Waals epitaxy," Science Advances, vol. 2, Article ID e1501882, 2016.

[18] H. Sun, Z. Wang, and Y. Wang, "Band alignment of twodimensional metal monochalcogenides MXs $(\mathrm{M}=\mathrm{Ga})$," AIP Advances, vol. 7, Article ID 095120, 2017.

[19] Q. Liu and A. Zunger, "Predicted realization of cubic Dirac fermion in quasi-one-dimensional transition-metal monochalcogenides," Physical Review X, vol. 7, Article ID 021019, 2017.

[20] L. Liu, Y. Feng, and Z. Shen, "Structural and electronic properties of h-BN," Physical Review B, vol. 68, Article ID 104102, 2003.

[21] A. B. Preobrajenski, M. A. Nesterov, M. L. Ng, A. S. Vinogradov, and N. Mårtensson, "Monolayer h-BN on lattice-mismatched metal surfaces: on the formation of the nanomesh," Chemical Physics Letters, vol. 446, no. 1-3, pp. 119-123, 2007.

[22] F. Schedin, A. K. Geim, S. V. Morozov et al., "Detection of individual gas molecules adsorbed on graphene," Nature Materials, vol. 6, no. 9, pp. 652-655, 2007.

[23] C. R. Dean, A. F. Young, I. Meric et al., "Boron nitride substrates for high-quality graphene electronics," Nature Nanotechnology, vol. 5, no. 10, pp. 722-726, 2010.

[24] A. K. Geim and I. V. Grigorieva, "Van der Waals heterostructures,” Nature, vol. 499, no. 7459, pp. 419-425, 2013.

[25] Y. Liu, N. O. Weiss, X. Duan, H.-C. Cheng, Y. Huang, and X. Duan, "Van der Waals heterostructures and devices," Nature Reviews Materials, vol. 1, pp. 1-17, 2016.

[26] K. S. Novoselov, A. Mishchenko, A. Carvalho, and A. H. Castro Neto, "2D materials and van der Waals heterostructures," Science, vol. 353, no. 6298, Article ID aac9439, 2016.

[27] M. Mahjouri-Samani, M. Tian, K. Wang et al., "Digital transfer growth of patterned 2D metal chalcogenides by confined nanoparticle evaporation," Acs Nano, vol. 8, no. 11, pp. 11567-11575, 2014.

[28] M. Mahjouri-Samani, R. Gresback, M. Tian et al., "Pulsed laser deposition of photoresponsive two-dimensional GaSe nanosheet networks," Advanced Functional Materials, vol. 24, pp. 6365-6371, 2014.

[29] D. J. Late, B. Liu, H. S. S. R. Matte, C. N. R. Rao, and V. P. Dravid, "Rapid characterization of ultrathin layers of chalcogenides on $\mathrm{SiO} 2 / \mathrm{Si}$ substrates," Advanced Functional Materials, vol. 22, no. 9, pp. 1894-1905, 2012.

[30] C. Ke, Y. Wu, G.-Y. Guo et al., "Tuning the electronic, optical, and magnetic properties of monolayer GaSe with a vertical electric field," Physical Review Applied, vol. 9, Article ID 044029, 2018. 
[31] P. Hu, Z. Wen, L. Wang, P. Tan, and K. Xiao, "Synthesis of few-layer GaSe nanosheets for high performance photodetectors," ACS Nano, vol. 6, no. 7, pp. 5988-5994, 2012.

[32] Q. Wang, K. Xu, Z. Wang et al., "Van der Waals epitaxial ultrathin two-dimensional nonlayered semiconductor for highly efficient flexible optoelectronic devices," Nano Letters, vol. 15, no. 2, pp. 1183-1189, 2015.

[33] N. Zhou, R. Wang, X. Zhou et al., "P-GaSe/N-MoS2 vertical heterostructures synthesized by van der Waals epitaxy for photoresponse modulation," Small, vol. 14, no. 7, Article ID 1702731, 2018.

[34] C.-W. Zhang and S.-S. Yan, "First-principles study of ferromagnetism in two-dimensional silicene with hydrogenation," The Journal of Physical Chemistry C, vol. 116, no. 6, pp. 4163-4166, 2012.

[35] R.-W. Zhang, C.-W. Zhang, W.-X. Ji et al., "Silicane as an inert substrate of silicene: a promising candidate for FET," The Journal of Physical Chemistry C, vol. 118, no. 43, pp. 2527825283, 2014.

[36] O. D. Restrepo, R. Mishra, J. E. Goldberger, and W. Windl, "Tunable gaps and enhanced mobilities in strain-engineered silicane," Journal of Applied Physics, vol. 115, Article ID 033711, 2014.

[37] C. Gang, L. Peng-Fei, and L. Zi-Tao, "Influence of strain and electric field on the properties of silicane," Chinese Physics B, vol. 22, Article ID 046201, 2013.

[38] S. Wang and J. Yu, "Tuning electronic properties of silicane layers by tensile strain and external electric field: a firstprinciples study," Thin Solid Films, vol. 654, pp. 107-115, 2018.

[39] K. L. Low, W. Huang, Y.-C. Yeo, and G. Liang, "Ballistic transport performance of silicane and germanane transistors," IEEE Transactions on Electron Devices, vol. 61, pp. 1590-1598, 2014.

[40] J. Shi, R. Tong, X. Zhou et al., "Temperature-mediated selective growth of MoS2/WS2 and WS2/MoS2 vertical stacks on $\mathrm{Au}$ foils for direct photocatalytic applications," Advanced Materials, vol. 28, no. 48, pp. 10664-10672, 2016.

[41] D. Ren, X. Tan, T. Zhang, and Y. Zhang, "Electronic and optical properties of GaN-MoS2 heterostructure from firstprinciples calculations," Chinese Physics B, vol. 28, Article ID 086104, 2019.

[42] J. Jiang, Q. Liang, S. Zhang et al., "Tuning the electronic and optical properties of graphane/silicane and fhBN/silicane nanosheets via interfacial dihydrogen bonding and electrical field control," Journal of Materials Chemistry C, vol. 4, no. 38, pp. 8962-8972, 2016.

[43] Z. Ben Aziza, H. Henck, D. Pierucci et al., "Van der Waals epitaxy of GaSe/graphene heterostructure: electronic and interfacial properties," ACS Nano, vol. 10, no. 10, pp. 9679-9686, 2016.

[44] K. D. Pham, C. V. Nguyen, H. T. T. Phung, H. V. Phuc, B. Amin, and N. N. Hieu, "Strain and electric field tunable electronic properties of type-II band alignment in van der Waals GaSe/MoSe2 heterostructure," Chemical Physics, vol. 521, pp. 92-99, 2019.

[45] A. Islam, J. Lee, and P. X.-L. Feng, “Atomic layer GaSe/MoS2 van der Waals heterostructure photodiodes with low noise and large dynamic range," ACS Photonics, vol. 5, no. 7, pp. 2693-2700, 2018.

[46] D. J. Late, B. Liu, J. Luo et al., "GaS and GaSe ultrathin layer transistors," Advanced Materials, vol. 24, no. 26, pp. 3549-3554, 2012.

[47] J. P. Perdew and Y. Wang, "Accurate and simple analytic representation of the electron-gas correlation energy," Physical Review B, vol. 45, no. 23, p. 13244, 1992.
[48] J. P. Perdew, K. Burke, and M. Ernzerhof, "Generalized gradient approximation made simple," Physical Review Letters, vol. 77, no. 18, p. 3865, 1996.

[49] M. Brandbyge, J.-L. Mozos, P. Ordejón, J. Taylor, and K. Stokbro, "Density-functional method for nonequilibrium electron transport," Physical Review B, vol. 65, Article ID 165401, 2002.

[50] J. M. Soler, E. Artacho, J. D. Gale et al., "The SIESTA method forab initioorder-Nmaterials simulation," Journal of Physics: Condensed Matter, vol. 14, no. 11, pp. 2745-2779, 2002.

[51] D. Chakraborty, K. Berland, and T. Thonhauser, "Introducing vdW-DF3-an accurate van der Waals density functional," Archives of the Bulletin of the American Physical Society, vol. 32, no. 9, 2020.

[52] P. T. T. Le, N. N. Hieu, L. M. Bui et al., "Structural and electronic properties of a van der Waals heterostructure based on silicene and gallium selenide: effect of strain and electric field," Physical Chemistry Chemical Physics, vol. 20, no. 44, pp. 27856-27864, 2018.

[53] H. L. Zhuang and R. G. Hennig, "Single-layer group-III monochalcogenide photocatalysts for water splitting," Chemistry of Materials, vol. 25, no. 15, pp. 3232-3238, 2013.

[54] B. P. Bahuguna, L. K. Saini, R. O. Sharma, and B. Tiwari, "Hybrid functional calculations of electronic and thermoelectric properties of GaS, GaSe, and GaTe monolayers," Physical Chemistry Chemical Physics, vol. 20, no. 45, pp. 28575-28582, 2018.

[55] G. G. Guzmán-Verri and L. C. Lew Yan Voon, "Band structure of hydrogenated Si nanosheets and nanotubes," Journal of Physics: Condensed Matter, vol. 23, no. 14, Article ID 145502, 2011.

[56] S. Wang and J. Yu, "Magnetic behaviors of 3d transition metal-doped silicane: a first-principle study," Journal of Superconductivity and Novel Magnetism, vol. 31, no. 9, p. 2789, 2018.

[57] B. Santu, P. Anup, P. Sougata, and S. Pranab, “Theoretical perspective on the photovoltaic performance of S,N-heteroacenes: an even-odd effect on the charge separation dynamics," The Journal of Physical Chemistry C, vol. 121, p. 2574, 2017.

[58] K. Moumita, S. Ritabrata, P. Sougata, and S. Pranab, "Tunable electronic structure of two-dimensional $\operatorname{MoX}_{2}(\mathrm{X}=\mathrm{S}, \mathrm{Se}) /$ $\mathrm{SnS}_{2}$ van der Waals heterostructures," The Journal of Physical Chemistry C, vol. 124, Article ID 21357, 2020.

[59] P. Anup, S. Sunandan, P. Sougata, and S. Pranab, "Pentacenefullerene bulk-heterojunction solar cell: a computational study," Physics Letters A, vol. 379, p. 1036, 2015.

[60] K. Moumita, R. Biplab, P. Sougata, and S. Pranab, "Engineering the electronic structure of tin sulfide nanoribbons: a computational study," The Journal of Physical Chemistry C, vol. 122, p. 5731, 2018.

[61] Z. Zhang, Z. Xie, J. Liu et al., "Band Alignment control in blue phosphorus $/ \mathrm{C}_{2} \mathrm{~N}$ van der Waals heterojunction by electric field," The Journal of Physical Chemistry, vol. 10, 2020.

[62] C. Xia, J. Du, M. Li et al., "Effects of electric field on the electronic structures of broken-gap phosphorene/ $\mathrm{SnX} \mathrm{X}_{2}(\mathrm{X}=\mathrm{S}$, Se) van der Waals heterojunctions," Physical Review Applied, vol. 10, Article ID 054064, 2018.

[63] K. D. Pham, N. N. Hieu, L. M. Bui et al., "Vertical strain and electric field tunable electronic properties of type-II band alignment C2N/InSe van der Waals heterostructure," Chemical Physics Letters, vol. 716, pp. 155-161, 2019.

[64] S. Wang and J. Yu, "Bandgap modulation of partially chlorinated graphene $(\mathrm{C} 4 \mathrm{Cl})$ nanosheets via biaxial strain and 
external electric field: a computational study," Journal of Applied Physics A, vol. 124, Article ID 487, 2018.

[65] K. Ren, S. Wang, Y. Luo et al., "Strain-enhanced properties of van der Waals heterostructure based on blue phosphorus and $\mathrm{g}-\mathrm{GaN}$ as a visible-light-driven photocatalyst for water splitting," RSC Advances, vol. 9, no. 9, Article ID 4816, 2019.

[66] S. Wang, U. M. Shoufie, and S. Riichiro, "Strain effect on circularly polarized electroluminescence in transition metal dichalcogenides," Physical Review Research, vol. 2, Article ID 033340, 2020.

[67] X. Tong, L. Fang, and R. Liu, "Strain-induced electronic properties of van der Waals heterostructures based on tin dichalcogenides," AIP Advances, vol. 9, Article ID 055324, 2019.

[68] X.-H. Li, B.-J. Wang, X.-L. Cai et al., "Strain-tunable electronic properties and band Alignments in $\mathrm{GaTe} / \mathrm{C}_{2} \mathrm{~N}$ heterostructure: a first-principles calculation," Nanoscale Research Letters, vol. 13, p. 300, 2018. 